\title{
The Parasitological, Immunological, and Molecular Diagnosis of Human Taeniasis with Special Emphasis on Taenia solium Taeniasis
}

\author{
Kabemba E. Mwape • Sarah Gabriël
}

Published online: 24 September 2014

(C) Springer International Publishing AG 2014

\begin{abstract}
Human neurocysticercosis, caused by the larval stage of the tapeworm Taenia solium, is an important neurological disorder reported as a major cause of epilepsy. An important risk factor for neurocysticercosis is the presence of human T. solium carriers who, upon open defecation, disseminate tapeworm eggs, which are infective to both humans and pigs. In the latter, infection also results in cysticercosis, with associated health and economic consequences. Control of T. solium therefore depends greatly on accurate detection and treatment of carriers. However, the currently available direct diagnostic tests depend on detection, in feces, of either parasite stages or parasite antigens and genetic material. The former are low cost but lack adequate sensitivity and specificity; the latter are too expensive to be routinely utilized in endemic communities. Indirect tests based on antibody detection may only show exposure and not active infection. An ideal diagnostic test should be one that is low cost and is able to quickly and reliably detect tapeworm carriers so that appropriate treatment can be prescribed in order to eliminate the source of infection. Such a test remains elusive. Efforts should, therefore, be directed at formulation of a test that is
\end{abstract}

K. E. Mwape $(\bowtie)$

Department of Clinical Studies, School of Veterinary Medicine,

University of Zambia, Lusaka, Zambia

e-mail: kemwape@yahoo.com

K. E. Mwape

e-mail: evans.mwape@unza.zm

K. E. Mwape

Department of Veterinary Tropical Diseases, Faculty of Veterinary

Sciences, University of Pretoria, Pretoria, South Africa

S. Gabriël

Department of Biomedical Sciences, Institute of Tropical Medicine,

Antwerp, Belgium not only sensitive and specific but also affordable for use in endemic countries.

Keywords Taenia solium diagnosis · Coproparasitology · Immunodiagnosis $\cdot$ Coproantigen ELISA $\cdot$ PCR

\section{Introduction}

Taenia solium, Taenia saginata, and Taenia asiatica are important tapeworms causing taeniasis in humans, who, as the natural definitive host of these cestodes, harbor the adult worm in the small intestine. Cattle serve as intermediate hosts for T. saginata, while pigs fulfill this role for T. solium and T. asiatica. Upon ingestion of infective eggs, intermediate hosts develop metacestode larval stages (also called cysticerci), resulting in bovine and porcine cysticercosis, respectively. Unlike the other two species, T. solium can also cause cysticercosis in humans. This occurs after inadvertent ingestion of T. solium eggs when metacestodes develop in organs and tissues, giving raise to cysticercosis, one of the most important parasitic conditions in humans. People acquire taeniasis following ingestion of undercooked pork or beef meat or viscera containing viable cysticerci. These develop into adult intestinal tapeworms, which, when mature, release proglottids (worm segments) laden with infective eggs. Proglottids may be passed relatively intact in feces, but frequently they disintegrate within the intestine, and so free eggs can be found in feces. The excreted eggs are immediately infective to the intermediate hosts [1], thus making the tapeworm carrier a fundamental key player in the transmission of cysticercosis. Garcia-Garcia et al. [2] demonstrated that the presence of tapeworm carriers in households is the main risk factor attributed to human cysticercosis. In the absence of sanitary facilities and/or adequate personal hygiene, these carriers become 
a major risk for members of their household and also community members [3].

In non-endemic countries, taeniasis is most likely to be imported by immigrant tapeworm carriers or people travelling to endemic areas, where they may acquire the infection through consumption of infected pork. Similarly, returning travelers may import cysticercosis if they ingest infective eggs from the contaminated environment, from food, or directly from carriers [4*0]. Additionally, migration of tapeworm carriers from rural to urban areas increases the risk of transmission of cysticercosis when there are poor environmental and social conditions [3].

While $T$. saginata has a more cosmopolitan distribution, T. solium is mostly reported in developing countries in Africa, Asia, and Latin America. T. asiatica, also known as Asian Taenia, is restricted to East Asian countries and has not been reported elsewhere in the world, including Africa [5]. T. solium endemicity in developing countries is associated with poverty, free-ranging pigs, and poor sanitary conditions, especially lack of latrines [1, 6, 7]. Many reports have documented T. solium infection in pigs in Africa, with prevalence rates as high as $64 \%$ [8].

As mentioned, the lodging of the metacestodes of T. solium in the central nervous system (CNS) results in neurocysticercosis (NCC), one of the most important neurological parasitoses in humans, and the main preventable cause of acquired epilepsy in endemic areas [9॰]. Unlike taeniasis, where symptoms are not of major clinical importance, the pathology caused by the establishment of $T$. solium metacestodes in the CNS may be responsible for a high disease burden and morbidity in endemic areas [1]. Unfortunately, the cysticercosis/ taeniasis disease complex remains a neglected tropical disease, with very little information on its current global burden. As a consequence, and as for many other parasitic zoonoses, its true burden still needs to be determined $[10,11]$. The current global burden of T. solium cysticercosis in terms of disability-adjusted life-years (DALYs) has been estimated at $2-5 \times 10^{6}$, an estimate comparable to those of other neglected parasitic zoonoses but less than those of the "big three" global infectious diseasesmalaria, HIV, and tuberculosis [12•]. Also, NCC is reported to account for about $30 \%$ of all reported cases of acquired epilepsy in endemic areas [13].

From an economic point of view, the presence of cysticerci in the specific intermediate hosts (i.e., cattle for T. saginata, and pigs for $T$. solium and T. asiatica), may be of great importance because of carcass condemnation in countries where meat inspection at the abattoir level is implemented $[1,11,12 \cdot]$. NCC is of great economic relevance, resulting from the cost of medical treatment and lost working days. A minimum estimate of the cost of admissions to hospital and wage loss for NCC in the USA (a non-endemic country) was US\$8.8 million annually, whereas in endemic countries such as Mexico and Brazil, treatment costs have been estimated at US\$89 million and US\$85 million, respectively [13].
Overall, T. solium has a higher public health impact than T. saginata, which mainly has economic implications for the meat industry [14]. Adult tapeworm infections are largely asymptomatic, though some people may experience abdominal discomfort, nausea, diarrhea, and loss of appetite, and in the case of $T$. saginata, itchiness of the anal area due to the actively migrating proglottids [15].

Taeniasis infections are increasingly being diagnosed in endemic areas of the world [1]. At the same time, there is growing recognition of $T$. solium as a serious emerging public health threat [16]. The data are, however, still very limited because of the lack of adequate surveillance, monitoring, and reporting systems. Compared with other helminth parasites, T. solium taeniasis tends to have a low prevalence, typically less than $1 \%$, even in endemic communities [17]. In fact, a prevalence $>1 \%$ is considered hyperendemic [18]. This is because in communities with inadequate sanitary infrastructure, a few tapeworm carriers have the potential to disseminate the infection to a great number of people and free-roaming pigs. Regions of endemicity have been identified [6, 19], with studies reporting prevalences ranging from $0.3 \%$ to $11.5 \%$ on coproparasitologic examination $[20-24,25 \bullet \cdot, 26 \bullet \cdot]$ and from $0.5 \%$ to $24.1 \%$ on coproantigen (copro- $\mathrm{Ag}$ ) enzyme-linked

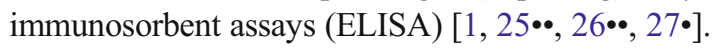

T. solium is considered a potentially eradicable parasite [28]. However, since the most affected areas are within developing countries, many ongoing challenges continue to hinder the implementation of control measures for this parasite. Obstacles that need to be overcome include lack of diagnostic facilities, inadequate or absent health infrastructure in rural areas, inaccessibility to health care and treatment with effective taeniacides, minimal cooperation between medical and veterinary services, and lack of knowledge about the parasite [1]. Several control options that target the various potential intervention points in the life cycle of the tapeworm have been described (Fig. 1). It is clear that control of taeniasis requires a multifaceted approach, as a single-intervention control program would not achieve the required results [29]. A control strategy that stands out is treatment of tapeworm carriers so as to remove the continued contamination of the environment in endemic areas. However, this strategy requires identification of such carriers, which has proven to be problematic because of the lack of low-cost and readily available diagnostic tools in resource-poor endemic areas.

This review looks at the currently available tools for taeniasis diagnosis and the strides made to date to improve them.

\section{Diagnosis of Taeniasis}

Diagnosis of taeniasis is mainly based on the search for parasitic material in feces [30]. Several tests have been developed, and each has its own advantages and disadvantages 
Fig. 1 Potential intervention points for preventing transmission of Taenia solium
Adult $T$. solium in humans

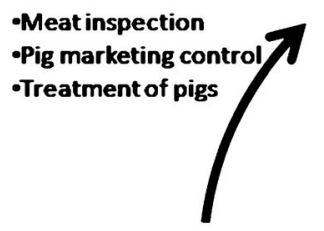

Cysticerci in pigs

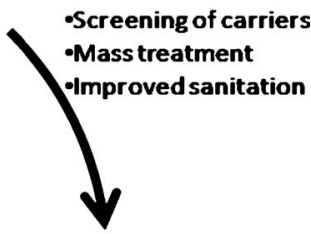

Eggs in the environment
(Table 1). Importantly, diagnostic gold-standard and costeffective tests are still lacking. The most widely used methods for taeniasis diagnosis are coproparasitological examination of feces to demonstrate the presence of Taenia spp. proglottids or eggs, and detection of specific coproantigens by an ELISA [31]. The possibility of detecting $T$. solium-specific antibodies in serum has also been demonstrated [32], and molecular methods have been reported.

\section{Self-Detection Tool for Tapeworm Carriers}

A common symptom associated with taeniasis is the expulsion of proglottids [33••], and carriers may report the presence of these in their feces or feel them in their undergarments [34]. However, while for T. saginata and T. asiatica, the proglottids may be spontaneously expelled independently of defecation,

Table 1 Currently available taeniasis diagnostic tests, with their main advantages and disadvantages

\begin{tabular}{lll}
\hline Test & Advantage & Disadvantage \\
\hline $\begin{array}{l}\text { Coproparasitologic } \\
\text { Self detection }\end{array}$ & Inexpensive & Unreliable \\
Microscopy & Highly specific & Low sensitivity \\
Immunological & & \\
Copro-Ag ELISA & Reasonably sensitive & Many false positives \\
Western blot & Highly specific & Many false positives \\
Molecular & & \\
PCR-based & Species differentiation & Very expensive \\
LAMP & Species differentiation & No field validation \\
\hline
\end{tabular}

Copro-Ag ELISA coproantigen enzyme-linked immunosorbent assay; $L A M P$ loop-mediated isothermal amplification; $P C R$ polymerase chain reaction the expulsion of $T$. solium proglottids is passive and they appear together with feces. The reliability of self-detection for taeniasis diagnosis has been evaluated [35-38], with sensitivity ranging from less than $50 \%$ in Honduras to over $80 \%$ in Sichuan, China [38, 39]. This wide variation has been explained by differences in the predominant Taenia species and the habits/customs of inhabitants in endemic areas [32]. Regardless, to be implemented as a reliable diagnostic tool, self-detection requires prior public health education campaigns [37].

\section{Coproscopic Examination of Feces}

The microscopic examination of stool samples (coproscopy) has remained the routine method for the diagnosis and identification of Taenia spp. eggs or proglottids to date. Direct wet mounts or concentration methods such as Kato-Katz and the formol-ether concentration technique [40] are widely used for the detection of Taenia spp. eggs in feces. The diagnostic sensitivity of these techniques, however, is not optimal, with reports ranging from 38 to $69 \%[41,42]$. Such low sensitivity is primarily due to the intermittent nature of egg release, which leads to an underestimation of the prevalence of taeniasis [43]. Allan et al. [44] reported that coproscopic studies from patients with active tapeworm infection are commonly negative because, firstly, eggs may not appear in feces every day, and secondly, eggs are not uniformly distributed in feces. For these reasons, the authors recommended collection of samples over a 3-day period. Further, if destrobilation (i.e., the breakage of gravid proglottids from the worm's body - the strobila) has led to a massive discharge of eggs, these may be absent from feces for up to several weeks thereafter, until more proglottids mature and become gravid [45]. In addition, the specificity of 
coproscopic methods is limited at the genus level because the eggs of these tapeworms are identical under light microscopy [1]. This is particularly relevant given the risks associated with T. solium infection [30].

Parasitological identification of human adult intestinal taeniids to species level relies on the recovery of gravid proglottids or scolices. This recovery is difficult because of the disintegration of the proximal end of the worm when modern cestocidal drugs are used [45]. Jeri et al. [46] improved the treatment method to obtain a recognizable tapeworm by using pre-niclosamide and post-niclosamide electrolytepolyethyleneglycol (PEG) salt purges to improve bowel cleaning and collection of the tapeworm scolex, making differentiation between T. saginata and T. solium easier. Nevertheless, since PEG has to be dissolved in $2 \mathrm{~L}$ of water, it might not be well accepted/perceived, especially in community studies.

Three morphological characteristics to distinguish T. solium from $T$. saginata were proposed by Verster [47] in a taxonomic review of the genus Taenia. These characteristics are the presence of an armed rostellum on the scolex, a three-lobed ovary, and the absence of a vaginal sphincter. Additionally, the number of uterine branches in gravid proglottids is an indicative but not absolute difference between the two Taenia species [48]. Fixation and staining of proglottids with Semichon's acetocarmine allows for identification of these differences, as does injection of liquid black ink through the genital pore. In addition to the absence (T. saginata) or presence (T. solium) of rostellar hooks on the scolex, Morgan and Hawkins [49] described a differential method based on the number of uterine branches in gravid segments. They reported that $T$. solium had between 8 and 14 unilateral uterine branches, whereas T. saginata had 15-24 branches. However, several authors have reported overlapping numbers, thus questioning the specificity of this method $[49,50]$.

The differential diagnosis of the adult worm causing taeniasis is very important for control purposes, but, in light of the factors explained above, diagnosis using morphological characteristics from parasite material is plagued with challenges.

\section{Parasite Coproantigen Assays}

Parasite coproantigens constitute specific products in the feces of the host that it is possible to detect using immunological tests. These products are associated with parasite metabolism, are independent of the presence of eggs or proglottids, and are reported to disappear from feces shortly after treatment $[30$, 51]. Coproantigens can also be detected as early as 2 weeks postinfection $[52 \bullet \bullet]$.

Several assays detecting Taenia coproantigens have been developed in different formats but all in the form of antigencapture ELISA, using polyclonal antibodies obtained from hyperimmunized rabbits with either adult worm somatic or excretory-secretory products $[25 \bullet \bullet, 53-56]$. These assays are reported to be genus specific and are independent of reproductive material (e.g., eggs). Furthermore, coproantigens are not detectable after treatment, and the antigens are stable in fecal samples [30], making the test very useful for early detection and evaluation of antiparasitic treatment efficacy in human T. solium taeniasis [51]. In epidemiological studies, the Copro-Ag ELISA is reported to detect around 2.5 times more cases of taeniasis than basic microscopy $[42,44]$.

The levels of sensitivity of these assays are dependent on the assay format (both microplate and dipstick formats have been used to date) and the quality of the rabbit sera used in their production (high-titer sera being better). Some studies have reported that these assays have specificity and sensitivity of $100 \%$ and $98 \%$, respectively $[31,55])$. Other studies in Guatemala and Peru have, however, recorded lower sensitivities [17, 56-58]. Using Bayesian analysis, a study by Praet et al. [59•] reported sensitivity and specificity of $85 \%$ and $92 \%$, respectively. The tests are genus specific; as such, T. saginata and T. solium infections cannot be differentiated. No cross-reactions with other infections, including Hymenolepis spp., Ascaris lumbricoises, Trichuris trichiura, hookworm and parasitic protozoa, have been identified [25••, 30]. To achieve species specificity, Guezala et al. [60] combined both polyclonal antibodies against $T$. solium adult whole worm extract and T. solium adult excretory-secretory proteins (ESP) in a hybrid sandwich ELISA format. This assay was reported to perform with $100 \%$ specificity and $95 \%$ sensitivity in the detection of T. solium carriers [60].

Though Allan and colleagues [41] already pointed out the presence of false positive results with the copro-Ag test in a field study conducted in Guatemalan communities, crossreactions with other parasites other than Taenia spp. have not been reported [30]. Nevertheless, potential nonspecific reactions of the polyclonal antibodies should be further investigated. In a study by Praet et al. [59], a sample that was T. saginata positive by copro-polymerase chain reaction (copro-PCR) was also copro-Ag positive, highlighting the nonspecificity of the copro-Ag test using polyclonal antibodies against adult $T$. solium. This calls for further improvements in the copro-Ag ELISA test, as the differential diagnosis of taeniasis has public health implications.

The copro-Ag ELISA is reported to detect immature tapeworm stages, and this could explain the higher number of copro-Ag ELISA-positive cases, compared with coproscopy (only detecting eggs and thus adult, gravid tapeworms) reported in studies that have used both tests. Further, studies that have used the copro-Ag ELISA test together with the molecular tests indicate that not all samples that are positive on copro-Ag ELISA are also positive on PCR $[26,58]$. In contrast with the copro-Ag ELISA, which is able to detect immature tapeworms, molecular-based tests are dependent on reproductive material such as eggs. This highlights the 
inadequacies of the latter to detect mature adult tapeworm carriers. Although based on one voluntarily self-infected subject, a study by Tembo and Craig [52••] reported that for T. saginata, coproantigens were detected 14 days postinfection, whereas proglottid patency occurred 86 days postinfection. If this is true for T. solium, then it could probably explain the higher number of copro-Ag ELISA positives compared with PCR reported in studies that have used both of these tests.

The rate at which tapeworms establish in the intestine following ingestion of cysticerci is not well known. It is generally assumed that only one tapeworm develops in a host (solitary worm). Competition between tapeworms of the same or different species, influencing their establishment, has been suggested by Conlan et al. [61]. Since people may consume pork meat infected with many cysts, potentially many of these can develop into adult worms within one host. However, an important proportion of infected individuals can harbor multiple tapeworms, as demonstrated in studies by Bustos et al. $(8.2 \%)$ [51] and Jeri et al. $(20 \%)$ [46]. It is also possible that some juvenile tapeworms are expelled before they reach maturity. Although cross-reactions have been demonstrated not to occur with the copro-Ag ELISA, additional studies to improve the test are required, and use of monoclonal antibodies to detect antigens in stool is suggested.

\section{Serological Diagnostic Assays}

Wilkins et al. [32] described T. solium-specific antigens to detect antibodies against adult $T$. solium in serum by Western blot analysis, with sensitivity and specificity rates of $95 \%$ and $100 \%$, respectively. Even though no cross-reactions were found in serum from individuals infected with $T$. saginata and other cestodes, including T. solium cysticercosis, one sample from a patient suffering from NCC but not harboring the intestinal worm tested positive [30]. The serological diagnosis of taeniasis has obvious advantages over the fecal-based methods (e.g., species specificity, avoidance of potential biohazard associated with collection and handling of fecal samples, and also the possibility of combining the test with other immunological assays in the diagnosis of cysticercosis). However, in treated individuals, antibodies remain detectable for a long time (period not yet established) and cause false positives [30, 62]. Further, as highlighted above, it is possible that after successful infection and initial establishment in the intestine, some tapeworms fail to progress into mature and gravid worms, consequently dying and being expelled from the body. In these situations, it is possible that individuals will remain positive for antibodies even when an actual infection cannot be demonstrated.

While these assays have been applied successfully as part of field research programs in endemic countries, issues such as cost and accessibility remain to be addressed if these tests are to be used routinely in these areas of the world [30]. The assays are also yet to be evaluated in large-scale field studies in endemic areas. For this reason, these tests are not yet commercially available for diagnosis but are available only for research purposes.

Handali et al. [63], described a rapid test method using recombinant proteins for immunodetection of taeniasis, which could be affordable, reliable, rapid, and easy to perform. Though feasible, the test still requires field evaluation and improvements of its sensitivity for taeniasis detection in endemic areas.

\section{Molecular Methods}

Molecular techniques have also been developed that allow species-specific tapeworm detection in feces and differentiation of collected parasite material [64-68, 69•]. Differentiation of human Taenia spp. by molecular assays is normally done on proglottids expelled from carriers after treatment [50, 70, 71]. In recent years, PCR tests for species-specific confirmation of Taenia spp. have been developed, based on the detection of parasite DNA in fecal samples (copro-DNA) [65], cysticerci $[65,72]$, or eggs present in the feces and on proglottids [65]. Several methods and loci have been used for differentiating Taenia spp. Gonzalez et al. [71] designated primers that have been used in multiplex PCR, giving differential detection between T. saginata and T. solium.

Mayta et al. [48] used PCR-restriction fragment length polymorphism (PCR-RFLP) to differentiate T. solium and T. saginata. They amplified the $3^{\prime}$ region of the $18 \mathrm{~S}$ and the $5^{\prime}$ region of the $28 \mathrm{~S}$ ribosomal gene (spacing the $5.8 \mathrm{~S}$ ribosomal gene) and used three restriction enzymes (AluI, DdeI or $M b o I$ ) for analysis of the PCR amplicons. Each enzyme gave a unique pattern for each species. In this assay, the primers amplified DNA from all cestodes, not only from Taenia spp.

Rodriguez-Hidalgo et al. [50] also differentiated Taenia spp. by PCR-RFLP using the $12 \mathrm{~S}$ rDNA but developed new primers to reduce the nonspecific amplification found when using field samples. They, however, also used DdeI as the restriction enzyme.

The major problem with PCR for DNA detection in stool samples has been that of sensitivity, owing to the presence of PCR inhibitors in stools [64, 73]. Mayta et al. [67] reported a nested-PCR assay targeting the Tso31 gene, which was developed for specific diagnosis of taeniasis due to T. solium. The specificity and sensitivity of the assay on archived samples were $97 \%$ (31/32) and $100 \%$ (123/123), respectively. Under field conditions, and using microscopy and/or Copro-Ag ELISA testing as the gold standards, the assay was $100 \%$ sensitive and specific.

Praet et al. [59•] reported a novel real-time PCR using T. solium-specific primers, TsolITS 145F and TsolITS 230R (Biolegio, Nijmegen, The Netherlands) and the Tsol_ITS_169Tq_FAM double-labeled probe (Biolegio) to 
detect T. solium-specific amplification. T. saginata-specific PCR primers and a detection probe were also chosen within the ITS1 sequence to amplify and detect $T$. saginata specifically. Using Bayesian analysis, this real-time PCR had sensitivity and specificity of $83 \%$ and $99 \%$, respectively. This study highlighted the importance of using Bayesian analysis in the estimation of diagnostic tests in light of the absence of a diagnostic gold standard for taeniasis.

The high sensitivity of species-specific detection of Taenia spp. is a major advantage of the copro-PCR test for the diagnosis of taeniasis $[59 \bullet, 64,65]$. However, molecular tools remain very expensive and unavailable in endemic areas. The current DNA extraction methods are too expensive to be used as a routine test, and many developing countries lack wellequipped laboratories needed for molecular tests [1], and this renders their use under field conditions unfeasible.

A report by Nkouawa et al. [68] described the development and evaluation of a loop-mediated isothermal amplification (LAMP) assay for differential diagnosis of infections with Taenia species. They demonstrated that the LAMP method was able to differentially detect Taenia species and had high sensitivity and specificity. The LAMP test is simple and highly cost effective compared with PCR, requiring simple inexpensive materials and equipment. The test was piloted on a limited number of clinical specimens, and therefore it requires field validation before it can be made available for routine differential diagnosis of taeniasis. If validated, the LAMP test has the potential to be used as an alternative and cost-effective tool for the detection of $T$. solium carriers globally.

\section{Conclusions}

The presence of $T$. solium tapeworm carriers in a community where open defecation is frequent leads to high human and porcine cysticercosis prevalences. A contaminated environment exposes individuals to repeated contact with the parasite. This has been demonstrated by incidence studies reporting high antibody seroconversion rates [25••, 74•]. Many of those individuals may end up with NCC and, as a result, may suffer from epilepsy for life. It is therefore the authors' view that the detection and treatment of carriers would be a great leap toward the control and elimination of taeniasis and cysticercosis in endemic communities. However, diagnostic deficiencies in the detection of adult-stage intestinal tapeworm carriers hamper control strategies that are based on detection and treatment of carriers [75]. Detection of eggs in feces is insensitive and nonspecific, while immunological and molecular tests still require refinement before they are made available to endemic communities at a relatively cheaper price than currently prevails. As highlighted in this review, taeniasis diagnosis is hindered by the lack of a diagnostic gold-standard test for T. solium detection.
From the public health point of view, it might be argued that taeniasis control could be approached in the same manner as soiltransmitted helminths: with mass drug administration (MDA), utilizing drugs such as niclosamide, which is reported to be both safe and efficacious. Regrettably, niclosamide is not readily available in many endemic countries, or it is not accessible to poor communities where the infection is prevalent. Further, since the taeniasis/cysticercosis disease complex remains a neglected problem, few resources are devoted to its control, if any at all.

The use of mass treatment has resulted in decreases in taeniasis and porcine cysticercosis prevalences in endemic areas $[18,29]$. However, since its effects last for only up to 2 years [76], MDA should be implemented for a number of years or should be combined with other control programs such as community education [77], vaccination of pigs [78-80], and improved veterinary control of pig slaughter [1]. As stated by Lightowlers [81], the future control of T. solium infections lies in an integrated approach, because a single control measure is unlikely to achieve effective and long-lasting control. Nevertheless, the reduction of environmental contamination with T. solium eggs by detection and treatment of carriers would be an important entry point. In resource-constrained settings, tapeworm carrier detection can be more cost-effective than MDA. Hence, low-cost, effective, quick, and easy to perform tests are urgently needed to detect these tapeworm carriers who are the cornerstone of taeniasis/cysticercosis transmission.

\section{Compliance with Ethics Guidelines}

Conflict of Interest Kabemba E. Mwape and Sarah Gabriël declare that they have no conflict of interest.

Human and Animal Rights and Informed Consent This article does not contain any studies with human or animal subjects performed by any of the authors.

\section{References}

Papers of particular interest, published recently, have been highlighted as:

- Of importance

.. Of major importance

1. Murrell KD. WHO/OIE/FAO guidelines for the surveillance, prevention and control of taeniasis/cysticercosis. Paris: World Health Organization for Animal Health (OIE); 2005. p. 2743.

2. Garcia-Garcia ML, Torres M, Correa D, et al. Prevalence and risk of cysticercosis and taeniasis in an urban population of soldiers and their relatives. Am J Trop Med Hyg. 1999;61:386-9.

3. Schantz PM, Moore AC, Munoz JL, et al. Neurocysticercosis in an Orthodox Jewish community in New York City. N Engl J Med. 1992;327:692-5.

4.• Yanagida T, Sako Y, Nakao M, et al. Taeniasis and cysticercosis due to Taenia solium in Japan. Parasitol Vectors. 2012;5:18. Even non- 
endemic countries are at risk of cysticercosis, as shown in this study highlighting the potential of $T$. solium carriers to pose a risk of infection to others.

5. Eom KS, Jeon HK, Rim HJ. Geographical distribution of Taenia asiatica and related species. Korean J Parasitol. 2009;47(Supplement):S115-24.

6. Phiri IK, Ngowi H, Afonso S, et al. The emergence of Taenia solium cysticercosis in Eastern and Southern Africa as a serious agricultural problem and public health risk. Acta Trop. 2003;87:13-23.

7. Sikasunge CS, Phiri IK, Phiri AM, et al. Risk factors associated with porcine cysticercosis in selected districts of Eastern and Southern provinces of Zambia. Vet Parasitol. 2007;143:59-66.

8. Dorny P, Phiri IK, Vercruysse J, et al. A Bayesian approach for estimating values for prevalence and diagnostic test characteristics of porcine cysticercosis. Int J Parasitol. 2004;34:569-76.

9. Carabin H, Ndimubanzi PC, Budke CM, et al. Clinical manifestations associated with neurocysticercosis: a systematic review. PLoS Negl Trop Dis. 2011;5:e1152. The ultimate effect of T. solium carriage is neurocysticercosis, which has various clinical manifestations with a considerable burden on those affected.

10. Carabin H, Budke CM, Cowan LD, et al. Methods for assessing the burden of parasitic zoonoses: echinococcosis and cysticercosis. Trends Parasitol. 2005;21:327-33.

11. Praet N, Speybroeck N, Manzanedo R, et al. The disease burden of Taenia solium cysticercosis in Cameroon. PLoS Negl Trop Dis. 2009:3:e406.

12. Torgerson PR, Macpherson CN. The socioeconomic burden of parasitic zoonoses: global trends. Vet Parasitol. 2011;182:79-95. The burden of T. solium cysticercosis entails the increasing need for its control.

13. Ndimubanzi PC, Carabin H, Budke CM, et al. A systematic review of the frequency of neurocyticercosis with a focus on people with epilepsy. PLoS Negl Trop Dis. 2010;4:e870.

14. Roberts T, Murrell KD, Marks S. Economic losses caused by foodborne parasitic diseases. Parasitol Today. 1994;10:419-23.

15. Gajadhar AA, Scandrett WB, Forbes LB. Overview of food- and water-borne zoonotic parasites at the farm level. Rev Sci Technol. 2006;25:595-606.

16. Muller R. Worms and disease: a manual of medical helminthology. London: Heinemann Medical Books Ltd; 1975.

17. Mafojane NA, Appleton CC, Krecek RC, et al. The current status of neurocysticercosis in Eastern and Southern Africa. Acta Trop. 2003;87:25-33.

18. Allan JC, Velasquez-Tohom M, Garcia-Noval J, et al. Epidemiology of intestinal taeniasis in four, rural, Guatemalan communities. Ann Trop Med Parasitol. 1996;90:157-65.

19. Cruz M, Davis A, Dixon H, et al. Operational studies on the control of Taenia solium taeniasis/cysticercosis in Ecuador. Bull World Health Organ. 1989;67:401-7.

20. Zoli A, Shey-Njila O, Assana E, et al. Regional status, epidemiology and impact of Taenia solium cysticercosis in Western and Central Africa. Acta Trop. 2003;87:35-42.

21. Newell E, Vyungimana F, Geerts S, et al. Prevalence of cysticercosis in epileptics and members of their families in Burundi. Trans $\mathrm{R}$ Soc Trop Med Hyg. 1997;91:389-91.

22. Asaava LL, Kitala PM, Gathura PB, et al. A survey of bovine cysticercosis/human taeniasis in Northern Turkana District, Kenya. Prev Vet Med. 2009;89:197-204.

23. Wohlgemut J, Dewey C, Levy M, Mutua F. Evaluating the efficacy of teaching methods regarding prevention of human epilepsy caused by Taenia solium neurocysticercosis in Western Kenya. Am J Trop Med Hyg. 2010;82:634-42.

24. Praet N, Kanobana K, Kabwe C, et al. Taenia solium cysticercosis in the Democratic Republic of Congo: how does pork trade affect the transmission of the parasite? PLoS Negl Trop Dis. 2010;4(9): e817.
25.• Mwape KE, Phiri IK, Praet N, et al. Taenia solium infections in a rural area of Eastern Zambia - a community based study. PLoS Negl Trop Dis. 2012;6(3):e1594. The use of improved diagnostic tests for taeniasis has led to the detection of many carriers. The authors of this study, however, also highlight the inadequacies of these tests in detecting active infections.

26.• Okello A, Ash A, Keokhamphet C, et al. Investigating a hyperendemic focus of Taenia solium in northern Lao PDR. Parasites Vectors. 2014;7:134. Though coproantigen-detecting tests detect more taeniasis positives, many such cases are negative on molecular tests.

27. Mwape KE, Phiri IK, Praet N, et al. The incidence of human cysticercosis in a rural community of eastern Zambia. PLoS Negl Trop Dis. 2013;7:e2142. The result of tapeworm carriage from environmental contamination is elucidated in this study, thus highlighting the need for detection and treatment of carriers to eliminate the contamination.

28. Centers for Disease Control and Prevention. Recommendations for the International Task Force for Disease Eradication. Morb Mortal Wkly Rep. 1993;42:28-38.

29. Sarti E, Rajshekhar V. Measures for the prevention and control of Taenia solium taeniasis and cysticercosis. Acta Trop. 2003;87:137-43.

30. Allan JC, Wilkins PP, Tsang VC, Craig PS. Immunodiagnostic tools for taeniasis. Acta Trop. 2003;87:87-93.

31. Allan JC, Avila G, Garcia-Noval J, et al. Immunodiagnosis of taeniasis by coproantigen detection. Parasitology. 1990;101(Pt 3): 473-7.

32. Wilkins PP, Allan JC, Verastegui M, et al. Development of a serologic assay to detect Taenia solium taeniasis. Am J Trop Med Hyg. 1999;60:199-204.

33.• Raoul F, Li T, Sako Y. Advances in diagnosis and spatial analysis of cysticercosis and taeniasis. Parasitology. 2013;140(13):1578-88. The available diagnostic tests for taeniasis are inefficient in the accurate diagnosis of the disease, as highlighted in this review.

34. Ito A, Li T, Chen X, et al. Mini review on chemotherapy of taeniasis and cysticercosis due to Taenia solium in Asia, and a case report with 20 tapeworms. Trop Biomed. 2013;30:164-73.

35. Sarti E, Schantz PM, Plancarte A, et al. Prevalence and risk factors for Taenia solium taeniasis and cysticercosis in humans and pigs in a village in Morelos, Mexico. Am J Trop Med Hyg. 1992;46:677-85.

36. Schantz PM, Cruz M, Sarti E, Pawlowski Z. Potential eradicability of taeniasis and cysticercosis. Bull Pan Am Health Organ. 1993;27: 397-403.

37. Flisser A, Vazquez-Mendoza A, Martinez-Ocana J, et al. Short report: evaluation of a self-detection tool for tapeworm carriers for use in public health. Am J Trop Med Hyg. 2005;72:510-2.

38. Li T, Ito A, Chen $\mathrm{X}$, et al. Usefulness of pumpkin seeds combined with areca nut extract in community-based treatment of human taeniasis in northwest Sichuan province. Acta Trop. 2012;124: $152-7$.

39. De Kaminsky RG. Albendazole treatment in human taeniasis. Trans R Soc Trop Med Hyg. 1991;85:648-50.

40. Ritchie LS. An ether sedimentation technique for routine stool examinations. Bull U S Army Med Dept. 1948;8:326.

41. Allan JC, Velasquez-Tohom M, Torres-Alvarez R, et al. Field trial of the coproantigen-based diagnosis of Taenia solium taeniasis by enzyme-linked immunosorbent assay. Am J Trop Med Hyg. 1996;54:352-6.

42. Sarti E. Taeniasis and cysticercosis due to Taenia solium. Salud Publica Mex. 1997;39:225-31.

43. Garcia HH, Gilman RH, Gonzalez AE, et al. Hyperendemic human and porcine Taenia solium infection in Peru. Am J Trop Med Hyg. 2003;68:268-75.

44. Allan JC, Velasquez-Tohom M, Fletes C, et al. Mass chemotherapy for intestinal Taenia solium infection: effect on prevalence in humans and pigs. Trans R Soc Trop Med Hyg. 1997;91:595-8. 
45. Gemmell MA, Matyas Z, Pawlowski Z, et al. Guidelines for surveillance, prevention and control of taeniasis/cysticercosis. Geneva: World Health Organisation; 1983. p. 49.

46. Jeri C, Gilman RH, Lescano AG, et al. Species identification after treatment for human taeniasis. Lancet. 2004;363:949-50.

47. Verster A. A toxonomic revision of the genus Taenia linnaeus, 1758S. str. Onderstepoort J Vet Res. 1969;36:3-58.

48. Mayta H, Talley A, Gilman RH, et al. Differentiating Taenia solium and Taenia saginata infections by simple hematoxylin-eosin staining and PCR-restriction enzyme analysis. J Clin Microbiol. 2000;38:133-7.

49. Morgan BB, Hawkins PA. Veterinary helminthology. Minneapolis: Burgess Publishing Company; 1949.

50. Rodriguez-Hidalgo R, Geysen D, Benitez-Ortiz W, et al. Comparison of conventional techniques to differentiate between Taenia solium and Taenia saginata and an improved polymerase chain reactionrestriction fragment length polymorphism assay using a mitochondrial 12S rDNA fragment. J Parasitol. 2002;88:1007-11.

51. Bustos JA, Rodriguez S, Jimenez JA, et al. T solium taeniasis coproantigen detection is an early indicator of treatment failure for taeniasis. Clin Vaccine Immunol. 2012;19(4):570-3.

52.• Tembo A, Craig P. Taenia saginata taeniasis: copro-antigen time-course in a voluntary self-infection. J Helminthol. 2014. doi:10.1017/ S0022149X14000455. The disease time course for taeniasis due to $T$. solium is not well established. The results of this study on T. saginata infection gives insights into the disease course and confirms the ability of the coproantigen ELISA to detect immature tapeworms.

53. Deplazes P, Eckert J, Pawlowski ZS, et al. An enzyme-linked immunosorbent assay for diagnostic detection of Taenia saginata coproantigens in humans. Trans R Soc Trop Med Hyg. 1991;85:391-6.

54. Maass M, Delgado E, Knobloch J. Detection of Taenia solium antigens in merthiolate-form preserved stool samples. Trop Med Parasitol. 1991;42:112-4.

55. Allan JC, Craig PS, Garcia-Noval J, et al. Coproantigen detection for immunodiagnosis of echinococcosis and taeniasis in dogs and humans. Parasitology. 1992;104(Pt 2):347-56.

56. Machnicka B, Dziemian E, Zwierz C. Detection of Taenia saginata antigens in faeces by ELISA. Appl Parasitol. 1996;37:106-10.

57. Garcia-Noval J, Allan JC, Fletes C, et al. Epidemiology of Taenia solium taeniasis and cysticercosis in two rural Guatemalan communities. Am J Trop Med Hyg. 1996;55:282-9.

58. Cabrera M, Verastegui M, Cabrera R. Prevalence of enteroparasitosis in one Andean community in the Province of Victor Fajardo, Ayacucho, Peru. Rev Gastroenterol Peru. 2005;25:150-5.

59. Praet N, Verweij JJ, Mwape KE, et al. Bayesian modelling to estimate the test characteristics of coprology, coproantigen ELISA and a novel real-time PCR for the diagnosis of taeniasis. Tropical Med Int Health. 2013;18:608-14. With the absence of a goldstandard test for taeniasis, evaluation of the performance of the available tests requires analyses that take into consideration prior information from other tests and from experts in the field.

60. Guezala MC, Rodriguez S, Zamora H, et al. Development of a species-specific coproantigen ELISA for human Taenia solium taeniasis. Am J Trop Med Hyg. 2009;81:433-7.

61. Conlan JV, Vongxay K, Fenwick S, et al. Does interspecific competition have a moderating effect on Taenia solium transmission dynamics in Southeast Asia? Trends Parasitol. 2009;25:398-403.

62. Ito A, Craig PS. Immunodiagnostic and molecular approaches for the detection of taeniid cestode infections. Trends Parasitol. 2003;19:377-81.

63. Handali S, Klarman M, Gaspard AN, et al. Development and evaluation of a magnetic immunochromatographic test to detect
Taenia solium, which causes taeniasis and neurocysticercosis in humans. Clin Vaccine Immunol. 2010;17:631-7.

64. Nunes CM, Lima LG, Manoel CS, et al. Taenia saginata: polymerase chain reaction for taeniasis diagnosis in human fecal samples. Exp Parasitol. 2003;104:67-9.

65. Yamasaki H, Allan JC, Sato MO, et al. DNA differential diagnosis of taeniasis and cysticercosis by multiplex PCR. J Clin Microbiol. 2004:42:548-53.

66. Nunes CM, Dias AK, Dias FE, et al. Taenia saginata: differential diagnosis of human taeniasis by polymerase chain reactionrestriction fragment length polymorphism assay. Exp Parasitol. 2005;110:412-5.

67. Mayta H, Gilman RH, Prendergast E, et al. Nested PCR for specific diagnosis of Taenia solium taeniasis. J Clin Microbiol. 2008;46: 286-9.

68. Nkouawa A, Sako Y, Nakao M, et al. Loop-mediated isothermal amplification method for differentiation and rapid detection of Taenia species. J Clin Microbiol. 2009;47:168-74.

69. Jeon HK, Yong TS, Sohn W, et al. Molecular identification of Taenia tapeworms by Cox1 gene in Koh Kong, Cambodia. Korean J Parasitol. 2011;49:195-7. Differentiation of Taenia tapeworms can be achieved by molecular methods.

70. Eom KS, Jeon HK, Kong Y, et al. Identification of Taenia asiatica in China: molecular, morphological, and epidemiological analysis of a Luzhai isolate. J Parasitol. 2002;88:758-64.

71. Gonzalez LM, Montero E, Puente S, et al. PCR tools for the differential diagnosis of Taenia saginata and Taenia solium taeniasis/cysticercosis from different geographical locations. Diagn Microbiol Infect Dis. 2002;42:243-9.

72. Yamasaki H, Nakao M, Sako Y, et al. DNA differential diagnosis of human taeniid cestodes by base excision sequence scanning thymine-base reader analysis with mitochondrial genes. J Clin Microbiol. 2002;40:3818-21.

73. Nunes CM, Lima LG, Manoel CS, et al. Fecal specimens preparation methods for PCR diagnosis of human taeniasis. J Inst Trop Med São Paulo. 2006;48:45-7.

74. Coral-Almeida M, Rodríguez-Hidalgo R, Celi-Erazo M, et al. Incidence of human Taenia solium larval infections in an Ecuadorian endemic area: implications for disease burden assessment and control. PLoS Negl Trop Dis. 2014;8:e2887. The result of tapeworm carriage from environmental contamination is again elucidated in this study, thus highlighting the need for detection and treatment of carriers to eliminate the contamination.

75. Schantz PM. Progress in diagnosis, treatment and elimination of echinococcosis and cysticercosis. Parasitol Int. 2006;55:S7-13.

76. Garcia HH, Evans CA, Nash TE, et al. Current consensus guidelines for treatment of neurocysticercosis. Clin Microbiol Rev. 2002;15:747-56.

77. Ngowi HA, Carabin H, Kassuku AA, et al. A health-education intervention trial to reduce porcine cysticercosis in Mbulu District, Tanzania. Prev Vet Med. 2008;85:52-67.

78. Flisser A, Gauci CG, Zoli A, et al. Induction of protection against porcine cysticercosis by vaccination with recombinant oncosphere antigens. Infect Immun. 2004;72:5292-7.

79. Gonzalez AE, Gauci CG, Barber D, et al. Vaccination of pigs to control human neurocysticercosis. Am J Trop Med Hyg. 2005;72: 837-9.

80. Assana E, Kyngdon CT, Gauci CG, et al. Elimination of Taenia solium transmission to pigs in a field trial of the TSOL18 vaccine in Cameroon. Int J Parasitol. 2010;40:515-9.

81. Lightowlers MW. Vaccines for prevention of cysticercosis. Acta Trop. 2003;87:129-35. 